\title{
A STUDY ON TUBAL PATENCY FOLLOWING MANAGEMENT OF TUBAL PREGNANCY
}

\author{
Surjeet Kumar Yadav' ${ }^{1}$ Renu Sangal ${ }^{2}$, Reeta Singh ${ }^{3}$, Harish Chandra Tiwari ${ }^{4}$, Neela Rai Sharma ${ }^{5}$, Reena Shrivastava ${ }^{6}$ \\ ${ }^{1}$ Resident, Department of Obstetrics and Gynaecology, BRD Medical College, Gorakhpur. \\ ${ }^{2}$ Associate Professor, Department of Obstetrics and Gynaecology, BRD Medical College, Gorakhpur. \\ ${ }^{3}$ Assistant Professor, Department of Obstetrics and Gynaecology, BRD Medical College, Gorakhpur. \\ ${ }^{4}$ Assistant Professor, Department of Obstetrics and Gynaecology, BRD Medical College, Gorakhpur. \\ 5 Professor, Department of Obstetrics and Gynaecology, BRD Medical College, Gorakhpur. \\ ${ }^{6}$ Professor, Department of Obstetrics and Gynaecology, BRD Medical College, Gorakhpur.
}

\begin{tabular}{l}
\hline ABSTRACT \\
BACKGROUND \\
A tubal ectopic pregnancy is one in which blastocyst implants in fallopian tube. Ectopic pregnancy accounts for $1-2 \%$ of total \\
pregnancies and $95 \%$ of them are tubal ectopic. It is an important cause of morbidity and mortality in the reproductive age group. \\
A woman with two ectopic pregnancies has a 10 -fold chance for another. \\
Aims \& Objectives- To study tubal patency following management of tubal ectopic pregnancy.
\end{tabular}

\section{MATERIALS AND METHODS}

A descriptive study of duration of 1 year, 50 cases from OB/GYN Dept., BRD Medical College, Gorakhpur.

\section{RESULTS}

Out of 50 cases, 30 cases were managed by surgery, 12 by methotrexate and 8 by expectant method. Free passage of dye was seen in contralateral tube $91 \%, 87 \%, 83.3 \%$ following methotrexate, expectant and surgical method respectively. There was statistically significant difference among groups. Free passage through the ipsilateral tube after MTX treatment was seen in $83 \%$ of the cases (10 out of 12). After expectant management, it was seen in $75 \%$ of the cases ( 6 out of 8 ). Difference was not significant.

\section{CONCLUSION}

Clinical treatment and surgery affect the contralateral tubal patency differently. Medical therapy should be preferred, if feasible.

\section{KEYWORDS}

Ectopic Pregnancy, Tubal Patency, Patients.

HOW TO CITE THIS ARTICLE: Yadav SK, Sangal R, Singh R, et al. A study on tubal patency following management of tubal pregnancy. J. Evolution Med. Dent. Sci. 2017;6(59):4353-4356, DOI: 10.14260/Jemds/2017/941

\section{BACKGROUND}

A tubal ectopic pregnancy is one in which blastocyst implants in fallopian tube. Ectopic pregnancy accounts for 1-2\% of total pregnancies and $95 \%$ of them are tubal ectopic pregnancy.(1) It is an important cause of morbidity and mortality in the reproductive age group. Mortality is related to severe haemorrhage from tubal rupture. The rupture rate ectopic pregnancy range from $20-35 \% .(2,3)$ A women with two ectopic pregnancies has a 10 -fold chances for another. Tubal rupture rate with ectopic pregnancy ranges from $20-35 \% .(4,5)$ Ectopic pregnancy is associated with reduced prospects of future fertility and subsequent live birth varied between $50 \%$ and $64 \%$.

Therefore, the assessment of tubal patency is of great importance in patients subsequent to a tubal ectopic pregnancy. Management of tubal ectopic pregnancy is done by either medical, salpingectomy or salpingostomy or by expectant method depending upon feasibility.

Financial or Other, Competing Interest: None.

Submission 17-06-2017, Peer Review 12-07-2017,

Acceptance 17-07-2017, Published 24-07-2017.

Corresponding Author:

Dr. Surjeet Kumar Yadav,

Old P.G Hostel, Room No.34

BRD Medical College,

Gorakhpur-273013,

Uttar Pradesh, India.

E-mail: ysurjeet@gmail.com

DOI: $10.14260 /$ jemds $/ 2017 / 941$
Tubal patency in the followup period after treatment can be assessed by hysterosalpingography.

Aim of our study is to know the tubal patency following management of tubal ectopic pregnancy.

\section{MATERIALS AND METHODS}

This descriptive study was conducted in Dept. of OB/GYN, BRD Medical College, Gorakhpur, India over a period of one year, July 2015 to June 2016.

A total of 50 patients of tubal ectopic pregnancy were studied. 30 cases underwent surgery, and 12 cases were managed by systemic treatment with methotrexate, 8 cases were observed by means of expectant management.

All 50 patients who took part in the study signed a written informed consent.

Out of these 50 cases, 29 cases were ruptured when they presented to us. After diagnosis and proper resuscitation, they were managed surgically. 21 cases were unruptured at the time of presentation. There were three groups of patients in our study on the basis of treatment given.

Inclusion criteria for surgery (Salpingectomy): Haemodynamically unstable patients with ruptured ectopic pregnancy, failure of clinical treatment, cross-sectional diameter of gestational sac is $>3.5 \mathrm{~cm}$, not desiring future pregnancy and failure of conservative surgery i.e. salpingostomy.

Inclusion criteria for Methotrexate treatment: Haemodynamically stable patients, increased or maintained level of beta-hCG level at 24 and 48 hours interval, no severe 
pain or persistent abdominal pain. Normal liver and kidney function tests. Cross-sectional diameter of gestational sac is < $3.5 \mathrm{~cm}$. Commitment to follow up until the ectopic pregnancy has resolved.

Patients who satisfied the selection criteria were treated with single dose of IM methotrexate, followed with regular measurement of beta-hCG on day 1, 4, 7. Patients who did not have a declining value of beta-hCG $>15 \mathrm{mIU} / \mathrm{mL}$ between days 4 and 7 were given $2^{\text {nd }}$ dose of MTX after 7 days of $1^{\text {st }}$ dose.

Patients with declining beta-hCG regularly followed up until their beta-hCG level was below $5 \mathrm{mIU} / \mathrm{mL}$.

The inclusion criteria for expectant management were: haemodynamic stability, decline of beta-hCG levels at 24 and 48-hour intervals, beta-hCG level $<3,000 \mathrm{mIU} / \mathrm{mL}$, crosssectional diameter of the mass $\leq 3.5 \mathrm{~cm}$, desire for future pregnancy and consent to participate in the study, through a document signed and approved by the Ethics Committee of the Institution. The patients were monitored weekly until the levels of beta-hCG were below $5 \mathrm{mIU} / \mathrm{mL}$. HSG was performed after 4 months only on patients in whom the expectant management was successful. The examination was performed after the tubal mass disappeared from ultrasound images, or when the patient decided to try for a new pregnancy.

Testing for tubal patency can be done by Hysterosalpingography or by laparoscopic chromopertubation. In our study, it was done by hysterosalpingography.

HSG was performed in immediate postmenstrual phase, 3 months after the successful medical treatment for the ectopic pregnancy (when the levels of beta-hCG became negative and the image of an extraovarian adnexal mass disappeared at transvaginal ultrasound). Before the procedure was done, pregnancy was ruled out, vaginal infections were treated and doxycycline prophylaxis was recommended to all patients. Because patients may experience cramping during the HSG, women were advised to take a non-steroidal antiinflammatory drug one hour prior to the procedure. The procedure was performed with a sterile technique, with a single-tooth tenaculum applied to align the cervical canal and uterine cavity. Approximately, $10 \mathrm{~mL}$ of water soluble contrast medium was injected through a cannula. Fluoroscopic examination was performed during the injection with patient repositioning as necessary. The examination was performed, read, and interpreted by a radiologist. HSG was demonstrated to have $65 \%$ sensitivity and $83 \%$ of specificity for tubal obstruction. Tubal cornual spasm can give false positive result. HSG is an excellent predictor of tubal patency but less effective at predicting normal tubal function or the presence of pelvic adhesion.

\section{Statistical Analysis}

Fisher exact test was applied to find out the significance of difference between two groups and the $p$ value $<0.05$ has been taken as a significant difference.

\section{RESULTS}

Tubal pregnancies are in the age group of 20-25 years.

29 cases $(58 \%)$ of tubal pregnancies were ruptured while 21 cases $(42 \%)$ were unruptured.
$36 \%$ cases had previous h/o of PID and 22\% cases had previous abortion and D\&C.

In the present study, $24 \%$ of the cases had h/o tuberculosis, and they were given antitubercular drugs along with ectopic pregnancy $\mathrm{m} / \mathrm{m}$.

\section{Distribution of Cases According to Management}

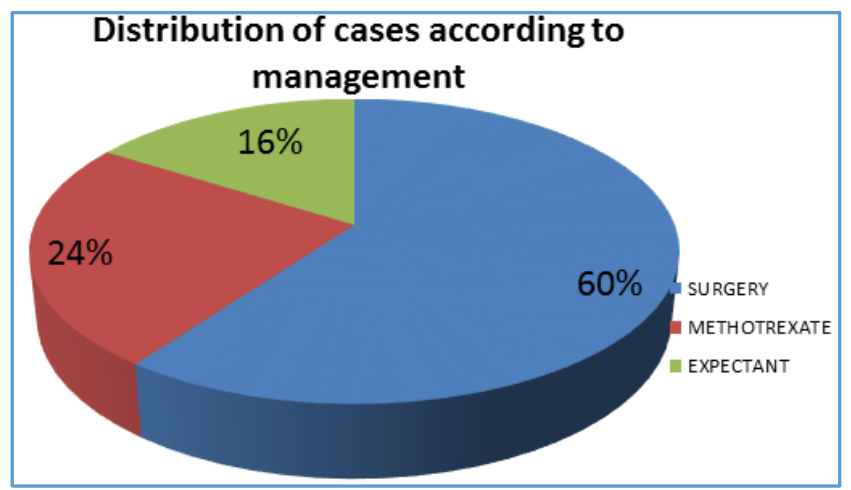

\begin{tabular}{|c|c|c|c|c|}
\hline $\begin{array}{c}\text { Hysterosal- } \\
\text { pingography/ } \\
\text { Diagnostic } \\
\text { Laparoscopy } \\
\text { SSG }\end{array}$ & $\begin{array}{c}\text { MTX } \\
\text { (\%) }\end{array}$ & $\begin{array}{c}\text { Expectant } \\
\text { Management } \\
\text { (\%) }\end{array}$ & $\begin{array}{c}\text { Salpingectomy/ } \\
\text { Salpingostomy } \\
\text { (\%) }\end{array}$ & $\begin{array}{c}\text { Total } \\
\text { (\%) }\end{array}$ \\
\hline Obstruction & 1 & 1 & 5 & 7 \\
$(9 \%)$ & $(13 \%)$ & $(16.67 \%)$ & $(14 \%)$ \\
\hline Patency & 11 & 7 & 25 & 43 \\
$(91 \%)$ & $(87 \%)$ & $(83.33 \%)$ & $(86 \%)$ \\
\hline Total & $\mathbf{1 2}$ & $\mathbf{8}$ & $\mathbf{3 0}$ & $\mathbf{5 0}$ \\
$(\mathbf{1 0 0 \% )}$ & $\mathbf{( 1 0 0 \% )}$ & $\mathbf{( 1 0 0 \% )}$ & $\mathbf{( 1 0 0 \% )}$ \\
\hline
\end{tabular}

Table 1. Post-treatment Tubal Patency seen via Hysterosalpingography on the Contralateral Tube Following Methotrexate (MTX), Expectant Management and Surgery

$(\chi 2=16.3, \mathrm{DF}=2, \mathrm{P}=<0.05)$

The contralateral tube patency levels were $91 \%, 87 \%$ and 83.3\%, following methotrexate treatment, expectant management and salpingectomy/salpingotomy, respectively (Table 1). There was significant difference found between the groups. This study shows that the contralateral tubal patency rate is better if managed through methotrexate.

\begin{tabular}{|c|c|c|c|}
\hline $\begin{array}{c}\text { Hysterosalpingography } \\
\text { Diagnostic laparoscopy } \\
\text { SSG } \\
\end{array}$ & $\begin{array}{l}\text { MTX n } \\
(\%)\end{array}$ & \begin{tabular}{|c|} 
Expectant \\
Management \\
n (\%) \\
\end{tabular} & $\begin{array}{l}\text { Total } \\
\text { n (\%) }\end{array}$ \\
\hline Obstruction & $\begin{array}{c}2 \\
(17 \%)\end{array}$ & $\begin{array}{c}2 \\
(25 \%)\end{array}$ & $\begin{array}{c}4 \\
(20 \%)\end{array}$ \\
\hline Patency & $\begin{array}{c}10 \\
(83 \%)\end{array}$ & $\begin{array}{c}6 \\
(75 \%)\end{array}$ & $\begin{array}{c}16 \\
(80 \%)\end{array}$ \\
\hline Total & $\begin{array}{c}12 \\
(100 \%)\end{array}$ & $\begin{array}{c}8 \\
(100 \%)\end{array}$ & $\begin{array}{c}20 \\
(100 \%)\end{array}$ \\
\hline \multicolumn{4}{|c|}{$\begin{array}{c}\text { Table 2. Post-treatment Tubal Patency seen via } \\
\text { Hysterosalpingography, SSG, or Diagnostic Laparoscopy } \\
\text { on the Ipsilateral Tube Following Methotrexate (MTX), } \\
\text { Expectant Management }\end{array}$} \\
\hline
\end{tabular}

$(\chi 2=0.208, D F=1, P=>0.05)$

Free passage through the ipsilateral tube after MTX treatment was seen in $83 \%$ of the cases (10 out of 12 ). After expectant management, it was seen in $75 \%$ of the cases ( 6 out of 8). P value was non-significant. Thus, the study shows that MTX treatment, expectant management have similar results 
post treatment patency. Ipsilateral tubal patency remains unaffected by the different methods of conservative management, i.e. MTX \& expectant management.

\section{DISCUSSION}

Out of 50 patients, 12 (24\%) cases had history of tuberculosis and they were managed with anti-tubercular treatment along with ectopic pregnancy treatment. Patients visiting to our department were usually referred from other centres and at the time of admission primary aim is survival of the patients, so the management is done accordingly. At the time of admission, it is difficult to record the tubal patency status of fallopian tube. Patients are first stabilised and treated with whatever best possible for the benefit of patients according to the patient's condition. After that patient is followed up after 3 months and then tubal patency is observed. Halbrecht (1957) noted a high incidence in patients who became pregnant following treatment for endometrial tuberculosis. ${ }^{(6)}$ Jeffcoat (2001) found unrecognised tuberculous salpingitis as an important cause and also in the recognised disease, which had been treated by antibiotics. This also indicates the prevalence of Koch's in the general population and its aetiological importance. It goes to prove the menace of tuberculosis in the present population studied. It is not only the reason for prolonged infertility, but also of the ectopic in case the patient finally conceives, thus endangering the patient's life.

Thus, the present series reinforces the fact that tuberculosis is India's biggest health problem and that it affects a major bulk of female population especially in not so developed region like the place of present study.

History of chronic PID was found in $18(36 \%)$ patients. It is in accordance with the cohort study in Lund (Sweden) in which much higher incidence of ectopic pregnancy was found in laparoscopically proven cases of PID compared with controls.(7)

There were 3 patients who had history of previous ectopic and again they developed tubal ectopic pregnancy. After one previous ectopic pregnancy, the chance of other approximates $10 \% .{ }^{(8)}$ Abnormal fallopian tube anatomy may be the cause of recurrent tubal ectopic. Prior sexually transmitted disease or other tubal infection, which can distort normal tubal anatomy, specifically, one episode of salpingitis can be followed by a subsequent ectopic pregnancy in up to $9 \%$ of women.(9) Similarly, peritubal adhesions subsequent to appendicitis, salpingitis or endometriosis may increase the risk of tubal pregnancy. These causes may be the reason for recurrent ectopic in our study.

Infertility per se as well as use of ART to overcome it, is linked to substantively increase risks for ectopic pregnancy.(10) In our study, there were 2 patients who had history of infertility which is in support of the above article. The tubal factor may be responsible for infertility as well as for ectopic pregnancy.

The difference between obstruction of the ipsilateral and contralateral tubes following clinical treatment may demonstrate the cases that suffer from sequelae of nonsurgical treatment. Obstruction of the contralateral tube gives important information about the case. This happened in $9 \%$ of the MTX cases and in $13 \%$ of the expectant management cases. These results suggest that before developing the ectopic pregnancy, some patients had an obstruction in the contralateral tube, probably caused by salpingitis, tuberculosis, etc. In the group that underwent surgery (salpingectomy or salpingotomy), contralateral tubal obstruction occurred in $16.67 \%$ of the cases. Obstruction of the contralateral tube was more frequent in the group that underwent surgery than in the clinical treatment groups. The explanation for these results is probably that surgery may give rise to more occurrences of adhesions because of the peritoneal factor, thereby resulting in infertility. Therefore, in the present study there was a significant difference between the groups in relation to contralateral tubal patency.

However, our sample size is very small and only few cases were managed by expectant and medical methods, the result may be influenced by sample size. It would be wise for more patients to be studied before accepting the equivalence between the treatments as a definite finding.

One multicentric trial compared multidose methotrexate protocol with laparoscopic salpingostomy and found no differences for tubal preservation and primary treatment success.(11)

\section{CONCLUSION}

Patency of ipsilateral tube after MTX treatment and expectant management was $83 \%$ and $75 \%$ respectively. Ipsilateral tubal patency remained unaffected by method of intervention.

The contralateral tube patency levels were $91 \%, 87 \%$ and $83.3 \%$ following methotrexate treatment, expectant management and salpingectomy/salpingotomy, respectively; i.e. methotrexate $t / t$ had better patency rate of contralateral tube. These results show that clinical treatment (MTX or expectant management) and surgery (salpingectomy) affect the tubal patency differently.

\section{REFERENCES}

[1] Pouly JL, Chapron C, Manhes H, et al. Multifactorial analysis of fertility after conservative laparoscopic treatment of ectopic pregnancy in a series of 223 patients. Fertil Steril 1991;56(3):453-60.

[2] Job-Spira N, Bouyer J, Pouly JL, et al. Fertility after ectopic pregnancy: first results of a population-based cohort study in France. Hum Reprod 1996;11(1):99104.

[3] Saxon D, Falcone T, Mascha EJ, et al. A study of ruptured tubal ectopic pregnancy. Obstet Gynecol 1997;90(1):46-9.

[4] Nordenskjo F, Ahlgren M. Risk factors in ectopic pregnancy: results of a population based case-control study. Acta Obstet Gynecol Scand 1991;70(7-8):575-9.

[5] Tuomivaara L, Ronnberg L. Ectopic pregnancy and infertility following treatment of infertile couples: a follow-up of 929 cases. Eur J Obstet Gynecol Reprod Biol 1991;42(1):33-8.

[6] Halbrecht I. Healed genital tuberculosis; a new etiologic factor in ectopic pregnancy. Obstet Gynaecol 1957;10(1):73-6. 
[7] Lund J. Early ectopic pregnancy; comments on conservative treatment. J Obstet Gynaecol Br Emp 1955;62(1):70-6.

[8] Ankum WM, Veen VDF, Hamerlynck JV, et al. Transvaginal sonography and human chorionic gonadotropin measurements in suspected ectopic pregnancy: a detailed analysis of a diagnostic approach. Hum Reprod 1993;8(8):1307-11.

[9] Westrom L, Joesoef R, Reynolds G, et al. Pelvic inflammatory disease and fertility: a cohort study of 1,844 women with laparoscopically verified disease and 657 control women with normal laparoscopic result. Sex Transm Dis 1992;19(4):185-92.
[10] Clayton HB, Schieve LA, Paterson HB, et al. Ectopic pregnancy risk with assisted reproductive technology procedures. Obstet Gynecol 2006;107(3):595-604.

[11] Hajenius PJ, Engelsbel S, Mol BW, et al. Randomised trial of systemic methotrexate versus laparoscopic salpingostomy in tubal pregnancy. Lancet 1997;350(9080):774-9. 